\title{
DEVELOPMENT OF A NEW DESIGN METHODOLOGY FOR LARGE SIZE PRODUCTS BASED ON DSM AND DFMA
}

\author{
B. Mora, I. Retolaza ${ }^{凶}$, M. A. Campos, A. Remirez, M. J. Cabello and F. Martinez \\ IKERLAN, Spain \\ $\triangle$ iretolaza@ikerlan.es
}

\begin{abstract}
A new design methodology for long life and large size (Ll-Ls) products called Design for Installation (DfI) is proposed. Ll-Ls products are usually made up of large parts that need to be assembled on field. The proposed methodology, based on adapted Design Structure Matrix (DSM) and Design for Manufacturing and Assembly (DfMA) methods, enables to optimize the design of a Ll-Ls product in order to reduce time and cost of the installation process. The new methodology works with a conceptual design of the product and the weight and size restrictions given by logistic factors as inputs.
\end{abstract}

Keywords: design for $x(D f X)$, design for installation, complex systems, modularisation, design structure matrix (DSM)

\section{Introduction}

In long life and large size products, the installation process has a major impact on the total cost (Steyn and Heerden, 2015). The work force needed to install the product in its final location, as well as the necessary tools for the transport and assembly of the product parts, are important factors to take them into account during the design process. The capacity of transport vehicles and lifting devices define the main dimensional, size and weight, restrictions of each part in which the product is divided (parts that can be defined as modules). Moreover, the quantity and complexity of the interfaces between the modules determine the amount of work required to assemble the product in its final location.

\subsection{State of the art}

Several references of cost reduction obtained by applying modularization and managing the work force and tools needed to install the product have been found. Mignacca et al. (2018) collected data related to time and cost savings achieved by applying modularization in engineering projects. As a summary of the study, an average of $37.7 \%$ in installation time reduction and a $15 \%$ reduction in the total cost of the project can be achieved (Mignacca et al., 2018). Fournier (2018) indicated that by applying modularization in engineering and architecture projects, a reduction of between $30 \%$ and $40 \%$ in on-site assembly time and a saving of up to $20 \%$ in installation cost can be obtained. Price et al. (2014) estimated that, applying modularization on a natural-gas processing plant project, it is possible to obtain reductions of up to $20 \%$ in installation time and of up to $15 \%$ in the total project cost. López et al. (2016) explained that in the construction of petrochemical facilities, using conventional construction methods can be up to $130 \%$ more expensive than using modular solutions, 
including risk factors related to climate, productivity, etc. Ou et al. (2018) used the construction of the Shanghai-Jiamin expressway as example to show the benefits of using prefabricated modules. A 50\% reduction in construction time and a $60 \%$ reduction in necessary workforce was achieved.

Until now, several design methodologies integrated in the Design for X (DfX) family has been developed (Kuo et al., 2001) (Figure 1). Each methodology in the DfX family focuses on specific characteristics of the designed product. Some of the most popular and most developed DfX methodologies are the Design for Assembly (DfA, focused on ease the assembly of products) and the Design for Manufacturing (DfM, focused on facilitate the manufacturing process of products) (Poli et al., 1992).
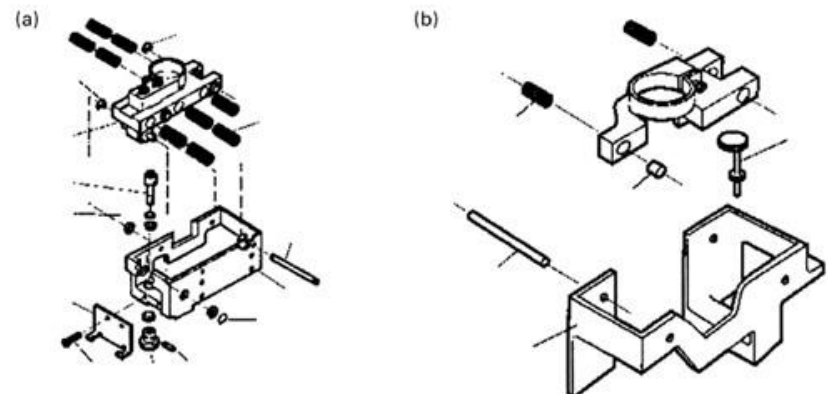

Figure 1. Reticle assembly. Comparison between traditional reticle design (left) and reticle design using DFA (right) (Kuo et al., 2001)

With the aim of adapting the traditional design methodologies to new requirements, variants of them have been created, such as Design for Automatic Assembly (DfAA, oriented to automated assembly of products by robots) (Nevelius and Deak, 2015) or Design for Additive Manufacturing (DfAM, oriented to product manufacturing by 3D printers) (Booth et al., 2017). However, a lack of a design methodology focused on reducing transportation and installation costs of large size products has been noticed.

No known DSM-based methodology focuses on the installation process of large equipment, using data of weight, dimensions and complexity of interfaces. In the work carried out by Rossi et al. (2019), this lack is mentioned in the conclusions, when the possibilities of future improvements of the methodology are listed.

With this lack as motivation, the Design for Installation methodology is proposed in this article.

\section{Design for Installation}

\subsection{Proposed methodology}

The Design for Installation (DfI) is a proposed design methodology included in the Design for X family (Figure 2). This methodology focuses on ease the transport and assembly of large size products in their final location with the aim of reducing installation time and costs, and consequently have a positive impact on the total cost of the project.

The type of products that the DfI focuses on has been defined as Long life and Large size (Ll-Ls). They consist of large size goods that are fixedly located in a specific place and need special devices (such as heavy vehicles and cranes) for their transport and installation processes. Some examples of Ll-Ls products are elevators, wind turbines, solar plants, pilot plants or petrochemical facilities.

The DfI uses the modularization as its main tool. The objective of the methodology is to get the optimal division of the product in modules in order to reduce the installation costs as much as possible. In order to facilitate the explanation of the DfI methodology, the following terminology has been created:

- Functional groups (FG): consist of the initial division of the product in parts. Usually, an FG is a part of the product that performs a specific function or group of functions. The definition of the FGs is flexible and is at the discretion of the user of the methodology.

- Installation groups (IG): consist of the division of the product in parts. They can be defined as assembly modules. An IG can match with an FG or it can be made up of some of them. 
- Installation tools (I-Tools): consist of the set of transport and installation devices necessary to assemble the IGs in their final location. The I-Tools define the weight and size restrictions on the IGs.

- Installation scenario (SC): consist of the set of elements that define the installation process of the product. The SC contains the product itself, with data about IGs configuration, and other data about the installation project context, including the installation tools, the installation site, human resources and special requirements.

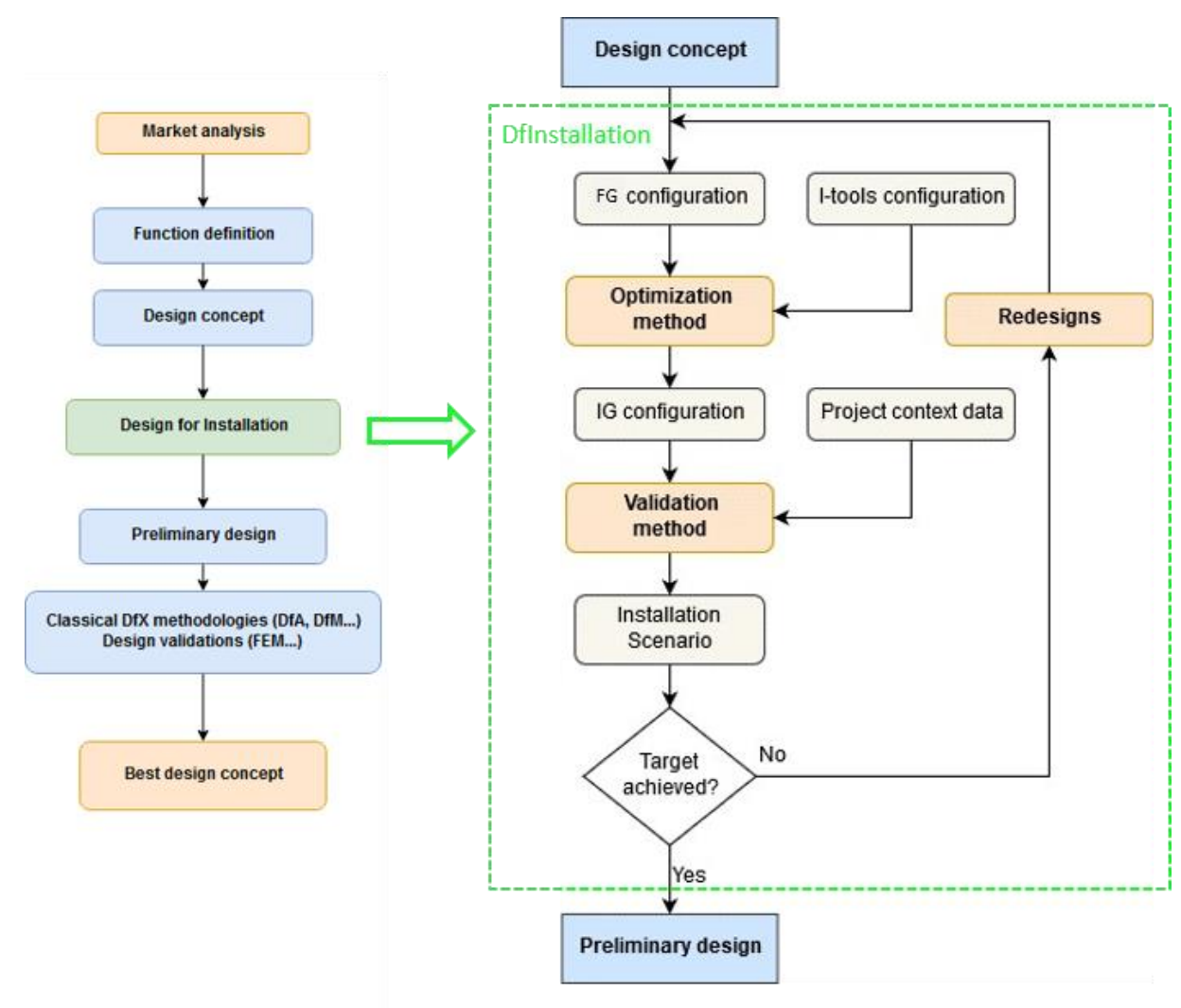

Figure 2. Flowchart of the Design for Installation methodology

To be effective, the DfI methodology should be applied in the early phases of project design, when the conceptual design of the product is already defined (and therefore the possible FGs and their characteristics are known) but before a complete preliminary design, because it may be conditioned by possible redesigns made during the application of the DfI.

It is clear that in the early design phase the available information can be very inaccurate, vague, unless the supplier has a lot of experience about the product, but it minimally serves to compare different alternatives before selecting a preliminary design.

The application of the methodology has an iterative nature. These are the main steps:

- Definition of the FGs (based on the conceptual design) and available installation devices (ITools) as inputs.

- Optimization: with these Inputs, optimization algorithm, through clustering, tries to group the product into modules that assume the lowest installation load. A configuration of IGs is obtained.

- Validation: when an IG configuration is obtained, it should be validated in order to confirm its feasibility. An installation process simulator is proposed as a method to perform the validation. With the proposed division in IGs of the product, and other available project data, a simulator can generate a complete model of the installation scenario (SC), through which it is possible to rate its viability. there are different commercial tools for this task, for example, Anylogic. 
The proposed IGs may involve changes in the conceptual design, which implies changes in the inputs. At this point, the process is repeated. The iterative process ends when the target of cost reduction is achieved.

If the results are deemed appropriate, the application of the DfI methodology is terminated and the design of the project continues. Otherwise if the results obtained are not considered adequate, the application must be repeated making any necessary changes, such as performing redesigns that affect functional groups, changing the installation media, etc.

To carry out redesigns in functional groups, the DfMA for Ll-Ls (Design for Manufacture and Assembly for Long life \& Large size products) methodology is used (Figure 3), developed in the study "Applying design methodologies to the use case of large products" (Ramos, 2018), conducted also in Ikerlan prior to this study.

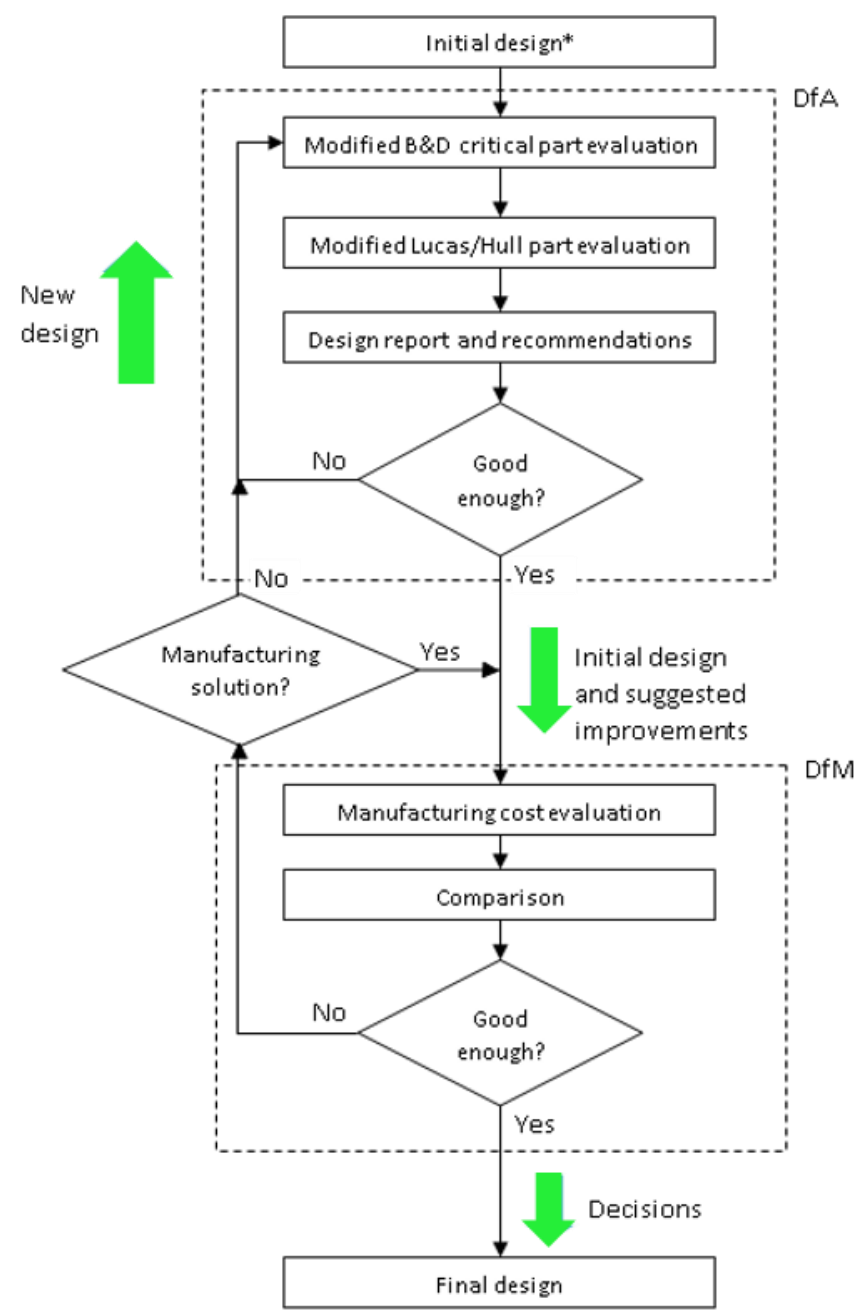

Figure 3. Application of the DfMA for LI-Ls methodology based on B\&D and L/Hull methods

This methodology, which starts from the existing DfM and DfA (Design for Manufacture and Design for Assembly), aims to reduce manufacturing and assembly costs in engineering projects. For the development of the DfMA for Ll-Ls, the appropriately modified Boothroyd-Dewhurst (Boothroyd, 1980; Boothroyd, 1987; Boothroyd, 1996) and Lucas-Hull (Lucas Engineering Systems Ltd., 1993; Masín, 2014) methods were used for application in large products.

This DfA / DfMA methodology has been developed and applied in some Ll-Ls product use case (Cabello et al., 2018; Remirez et al., 2019).

DfMA for Ll-Ls is proposed as an integrated tool within the DfI methodology to perform redesigns in functional groups. Some of the redesigns obtained may not have a direct impact on interface loads, but they may involve improvements in other aspects (such as material cost or factory assembly cost). 


\subsection{Design Structure Matrix}

A Design Structure Matrix (also known as Dependency Structure Matrix) is a representation of a system in the form of a squared matrix. The objective of a DSM is to collect the interactions between the different elements of the system. Each element corresponds both to a file (i) and a row (j) of the matrix. Each position of the matrix $(i, j)$ stands for the possible interaction between two elements of the system. If an interaction exists, a value is assigned to this position; otherwise, the position remains blank. Positions in the diagonal of the matrix $(i=j)$ represent the interactions of the elements with themselves, which most times does not suppose interesting information. Due to it, diagonal positions are not usually used.

A DSM always has an equivalent graph (Figure 4). Matrices whose equivalent graphs are directed, are non-symmetrical. Instead, matrices whose equivalent graphs are undirected, are symmetrical.

Binary DSM are those in which their interaction values only can be 1, representing an existing interaction. Non-binary DSM are those in which their interaction values are variable, being possible to be discrete or real values.

\begin{tabular}{|c|c|c|}
\hline FG1 & & \\
\hline $1-2$ & FG2 & \\
\hline $1-3$ & $2-3$ & FG3 \\
\hline
\end{tabular}

\begin{tabular}{|c|c|c|c|c|c|}
\hline $\begin{array}{l}\text { weight } \\
\text { width }\end{array}$ & $\begin{array}{l}\text { lenght } \\
\text { height }\end{array}$ & & & & \\
\hline $\begin{array}{l}\text { int. load } \\
\text { comb. factor W }\end{array}$ & $\begin{array}{l}\text { comb. factor } \mathrm{L} \\
\text { comb. factor } \mathrm{H}\end{array}$ & $\begin{array}{l}\text { weight } \\
\text { width }\end{array}$ & $\begin{array}{l}\text { lenght } \\
\text { height }\end{array}$ & & \\
\hline $\begin{array}{c}\text { int. load } \\
\text { comb. factor W }\end{array}$ & $\begin{array}{l}\text { comb. factor } \mathrm{L} \\
\text { comb. factor } \mathrm{H}\end{array}$ & $\begin{array}{l}\text { int. lood } \\
\text { comb. factor } W\end{array}$ & $\begin{array}{l}\text { comb. factor L } \\
\text { comb. factor } \mathrm{H}\end{array}$ & $\begin{array}{l}\text { weight } \\
\text { width }\end{array}$ & $\begin{array}{l}\text { lencht } \\
\text { height }\end{array}$ \\
\hline
\end{tabular}

Figure 4. Structure of the data in the DSM-DFI matrix. Sub-matrices in green correspond to functional groups, and sub-matrices in orange correspond to interactions between them

The Design Structure Matrix as a management method was studied for the first time by Steward in 1981. He proposed the DSM as an alternative to graphs for order tasks in an optimal way according to their interdependencies (Steward, 1981). In 1989, the NASA started to use the DSM as a method to manage their projects. They also developed an algorithm (the DeMAID/GA: Design Manager's Aid for Intelligent Decomposition/Genetic Algorithm) that was able to reorder the elements of the matrix with the aim of finding the optimal organization of the tasks and work teams for their projects (Rogers, 1996). The MIT researchers Pimmler and Eppinger (1994), with the collaboration of the Ford Motor Co., applied the DSM method to the product design for first time. Gutierrez (1998) developed a stochastic clustering algorithm based on the work of Pimmler and Eppinger. This algorithm was improved by Thebeau (2001). Since then, several DSM methodologies for multiple applications have been created. The work carried out by Rossi et al. (2019), which consisted in a DSM-based methodology for modularization of production plants, is an example of a specific application of DSM-based modularization.

The main reason why the DSM as a system representation method is preferable to others (such as graphs) is its numeric matrix format. Due to it, several kinds of algorithms can work directly with DSM data.

\subsection{DSM-DFI}

The DSM-DFI (standing for Design Structure Matrix-Design for Installation) is a variation of the DSM, specifically developed for its use in the Design for Installation methodology. A DSM-DFI matrix represents a complete Ll-Ls product, containing numerous information about it. The main feature of the DSM-DFI matrix is its double dimension: two rows and two columns correspond to each element of the system. Considering this, the DSM-DFI matrix can be understood as a large matrix made up of 2x2 sub-matrices. Every sub-matrix stands for an element of the system (in the case of being located on the diagonal of the main matrix) or an interaction between two elements (in the case of being located out of the diagonal of the main matrix).

Every element of the system (understanding the system as the whole product) corresponds to a functional group (FG) and contains four values: the weight and three dimensions of that FG. In submatrices corresponding to the interactions between elements, four values are also stored: the interaction load and three combination factors. 
The interaction load is a dimensionless value that represents the workload required to assemble the two elements on-site. The combination factors are auxiliary values that permit to obtain the final dimensions of a group of elements. The way these factors work is explained with the following example: if there is two parts (functional groups), $2 \mathrm{~m}$ long each, but the assembly of the two parts has a length of $3.5 \mathrm{~m}$, the length comb. factor of the interaction is $-0.5 \mathrm{~m}$. There is a combination factor for each of the three dimensions.

The DSM-DFI matrix is always symmetrical because the interfaces it contains represent physical unions between parts of a product (Figure 5). The interface between a part A and a part B is the same that the interface between a part B and a part A. Therefore, to avoid redundancies, only one of the halves of the DSM-DFI matrix is used.

\begin{tabular}{|c|c|c|c|c|c|c|c|}
\hline 550 & 2.2 & \multirow{2}{*}{\multicolumn{2}{|c|}{1}} & & & & \\
\hline 1.5 & 1.1 & & & & & & \\
\hline 50 & -0.5 & 750 & 2.5 & & & & \\
\hline \multirow[t]{6}{*}{-0.3} & 0 & 1.2 & 1 & & & & \\
\hline & & 70 & -0.4 & 960 & 3 & \multirow{2}{*}{\multicolumn{2}{|c|}{2}} \\
\hline & & -0.2 & 0 & 2 & 1.8 & & \\
\hline & & & & 60 & -0.2 & 600 & 2 \\
\hline & & & & 0 & -0.3 & 1.2 & 1 \\
\hline & & \multicolumn{2}{|c|}{ Weigth (kg) } & Length ( $m$ ) & \multicolumn{2}{|c|}{ Width (m) } & Heigth (m) \\
\hline \multicolumn{2}{|c|}{ cluster 1} & \multicolumn{2}{|c|}{1300} & 4,2 & \multicolumn{2}{|c|}{2,4} & 2,1 \\
\hline \multicolumn{2}{|c|}{ cluster 2} & \multicolumn{2}{|c|}{1560} & 4,8 & \multicolumn{2}{|c|}{3,2} & 2,5 \\
\hline \multicolumn{2}{|c|}{ w\&s restrictions } & \multicolumn{2}{|c|}{1600} & 5 & \multicolumn{2}{|c|}{3,5} & 2,8 \\
\hline
\end{tabular}

Figure 5. Example of a clustered DSM-DFI matrix

\subsubsection{Interaction load}

The objective of this article is not to develop a complete calculation method for the interaction load. Despite this, a proposed method for calculate this value is showed as an example. The interaction load must be able to valuate so that a precise comparison can be made between the different interactions of the FGs. Therefore, is not important the value of the interaction load itself, but the fact that the different interaction loads are comparable to each other. Thus, the criteria used to evaluate the different interactions must be equivalent.

During the development of the DfI methodology has been noticed a lack of public information to create a precise method to calculate the interaction load values. Due to this, the user of the DfI methodology is encouraged to develop his own criteria to evaluate the interaction loads based on the data available to him. As an example, the expression (1) shows a method to calculate the interaction load for fixed joints.

$$
\text { I_load_f }=1000 * L * W * \Pi \mathrm{i}(\mathrm{Ki})
$$

The L stands for the length of the joint, the $\mathrm{W}$ stands for the weight of the part to assembly and $\mathrm{Ki}$ consist of a set of penalties that assess the interaction depending on the type of joint and other characteristics (such as required alignment, tightness, detachability, etc.). The output means the assembly complexity between Functional Groups (FGs); the higher the value, the more complex the assembly. For example, in a welded joint, the bigger the length the higher the time and welding cost; the bigger the weight of the part the longer handling and positioning time, etc.

This method has been chosen because of its simplicity and its objectivity at the time of evaluating a physical interaction based on the complexity of assembling it on-site. For this, the method first valuates the interaction using the main factors that determine the difficulty of assembling the interaction (weight and length) and later the initial value is adjusted with the use of penalties, which represent other characteristics of the interaction, with the intention to achieve an accurate definition of its assembly complexity. A multiplier number can be added if is preferred to work with major or minor values. 
Equivalent methods can be developed to evaluate other types of interactions, such as mobile joints, electrical unions, etc. Interactions with null load value can be used to represent provisional unions between FGs, as for example, provisional packs of FGs to facilitate their transport.

The values assigned to the penalties are difficult to define due to the lack of public data about labour costs. Despite this, some penalties based on estimates and some sources have been defined. The Table 1 shows a group of joint type cost and detachability penalties as an example.

Table 1. Example of penalties for fixed joints

\begin{tabular}{|l|c|c|}
\hline \multicolumn{1}{|c|}{ Joint type } & Cost pen. (Kc) & Detachability pen. (KD) \\
\hline Welded & 1 & 1 \\
\hline Screwed & 0.83 & 0.5 \\
\hline Riveted & 0.84 & 0.8 \\
\hline Clinched & 0.62 & 1 \\
\hline Adhesive & 0.9 & 1 \\
\hline
\end{tabular}

Interactions with null load value can be used to represent provisional unions between FGs, as for example, provisional packs of FGs to facilitate their transport. It is not recommended to mix interactions with load values and interactions with null values in the same matrix, because they are not equivalent and can generate non optimal clustering results.

For undesired interactions (interactions between FGs that the user does not want to joint in the same installation group) negative interaction load values can be used.

\subsubsection{Clustering the DSM-DFI}

The main target of the DSM-DFI matrix is to find the optimal division of it into clusters. Clustering a DSM-type matrix is the process in which the elements of the matrix are grouped in packs, with the criterion of joining the elements that share high-value interactions. In the case of the DSM-DFI, every cluster obtained from the clustering process matches with an installation group (IG).

The main intention of the Design for Installation methodology is to obtain the optimal division into assembly modules or IGs of a large size product, in order to reduce installation time and costs. Taking this into account to perform the clustering of the DSM-DFI matrix two requirements are considered: intracost maximization and weight and size restrictions. The aim of the clustering process can be summed up by finding the largest possible clusters that fit the weight and size restrictions imposed by the I-Tools.

In order to manage the interactions between elements during the clustering process, the following concepts are used:

- Intracost: sum of all interaction loads enclosed by clusters.

- Extracost: sum of all interaction loads outside of clusters.

- Totalcost: sum of all interaction loads in the DSM-DFI.

- Optimization ratio: ratio between intracost and totalcost.

To proceed to perform the DSM-DFI clustering, it is necessary to use a computer algorithm. Until now, several clustering algorithms have been developed. Stochastic algorithms were developed by Gutierrez (1998), Thebeau (2001) and Borjesson and Hölttä-Otto (2014). This type of algorithms works by generating random clusters and comparing them, choosing those that maximize the intracost. Later, some alternatives were adapted to the cluster search, such as genetic algorithms or Cuckoo search algorithms (Wahdan et al., 2016). The results delivered by a clustering algorithm consist of an optimal group of clusters (corresponding to IGs) to reduce installation time and costs.

A sequencing algorithm is capable of reorder the rows and columns of the DSM-DFI matrix. The rearrangement brings together the FGs that share high value interactions. It is highly recommended to apply a sequencing algorithm before applying the clustering algorithm, because if the FGs that share high value interactions are not close, the clustering algorithm may not obtain optimal results. An example of a sequencing algorithm adapted to DSM matrices is the genetic algorithm DeMAID/GA developed by the NASA in 1989. 


\begin{tabular}{|c|c|c|c|c|c|c|c|}
\hline 550 & 2.2 & \multirow{2}{*}{\multicolumn{2}{|c|}{1}} & & & & \\
\hline 1.5 & 1.1 & & & & & & \\
\hline 50 & -0.5 & 750 & 2.5 & & & & \\
\hline \multirow[t]{5}{*}{-0.3} & 0 & 1.2 & 1 & & & & \\
\hline & & 70 & -0.4 & 960 & 3 & & \\
\hline & & -0.2 & 0 & 2 & 1.8 & 2 & \\
\hline & & & & 60 & -0.2 & 600 & 2 \\
\hline & & & & 0 & -0.3 & 1.2 & 1 \\
\hline
\end{tabular}

\begin{tabular}{|l|c|}
\hline intracost & 110 \\
extracost & 70 \\
totalcost & 180 \\
opt. ratio & $61 \%$ \\
\hline
\end{tabular}

Figure 6. Cost values of a clustered DSM-DFI matrix

\subsection{Advantages of DSM-DFI over other DSM-based methods}

Until now, many methods of management and modularization of projects based on DSM have been developed, with different approaches. The methodology developed by Pimmler and Eppinger (1994), pioneer in the application of DSM in product design, evaluates qualitatively the interactions between functional groups. The obtained clusters have the purpose of optimizing product design tasks, based on jointly developing those parts that share more interactions.

Some DSM-based methodologies developed later define themselves as "modularization methodologies". These methodologies include a division of the product into functional groups of a product and usually evaluate the interactions in a qualitative way. It is often not specified whether the resulting clusters have application in the product design tasks or in the design of the product. Some examples are the methodologies developed by Sharman and Yassine (2007) and Borjesson and Hölttä-Otto (2012).

The method developed by Rossi et al. (2019) proposes the use of several DSM: a base matrix that collects the interactions and other dimensionally equivalent matrices that collect data about constraints, costs and different penalties according to the criteria used for modularization. This methodology, focused on the modularization of production plants, is one of the most specific and complete DSM-based modularization methodologies developed until today.

However, no known DSM-based methodology focuses on the installation process of large equipment, using data of weight, dimensions and complexity of interfaces. This is the main difference of the DSM-DFI methodology from other DSM-based methodologies. Weight and size of the modules are not data used by any of the known DSM methods. In the article published by Rossi et al. (2019), this lack is mentioned in the conclusions, when the possibilities of future improvements of the methodology are listed. The most remarkable qualities of the DSM-DFI are listed below:

- The DSM-DFI matrix has more data storage capacity than other DSM matrices thanks to its double dimension and the capacity of store data in the diagonal positions (most DSM matrices do not use these positions). Several values are stored for each FG and each interaction.

- The combination factors are an own feature of the DSM-DFI method. Due to them, the real dimensions of any possible cluster of the matrix (corresponding to a possible assembly module) can be calculated.

- The DSM-DFI uses quantitative interaction loads; these loads represent physical magnitudes such as weight, size, interaction load... In some way they are values that represent physical concepts. Thanks to this, it has the capacity to be more objective at the time of evaluate the amount of work needed to assemble on-site every possible installation group. Other matrix-based methods, such as the Pimmler and Eppinger's (1994) method, evaluate the interactions between elements using values that do not represent any physical magnitude but simply a value, a note, which implies more subjectivity. 


\section{Conclusions and future work}

\subsection{Conclusions}

A new design methodology focused on ease the installation process of large size products has been developed, covering a lack noticed in the Design for X family.

After a state-of-the-art research, it has been concluded that there are several modularization methodologies that can be used as basis to develop the Design for Installation methodology. Most of those modularization methodologies are DSM-based. Due to this, the Design Structure Matrix has been used as a basis in the methodology proposed in this article. A variant of the DSM matrix, called DSM-DFI and focused on the installation of large size goods, has been proposed. The DSM-DFI can store more data than others DSM matrices. The possibility of working with the weight and dimensions of the elements and quantitatively evaluate the interactions between elements are other of the main characteristics of the DSM-DFI. The output obtained after the application of sequencing and clustering algorithms can be used to improve the transport and assembly process of a large size goods, with the aim of reducing installation time and costs.

\subsection{Future work}

Further work includes the application of the methodology in diverse case studies, with the objective of test its robustness and capacity of obtain improvements in different kinds of products.

In addition, some points of the methodology need further research. The DSM-DFI matrix must be improved. The calculation method of the interaction loads can be improved by providing more information about the time and costs needed to assemble different types of interactions, which would allow obtaining more accurate interaction penalties. The incorporation of data about costs of the installation tools would also allow the method to achieve better accuracy. The application of a simulator (such as discrete-event or agent-based simulators) must be validated. A simulation software must be chosen and a case study, including the simulation of a complete installation scenario, must be carried out.

\section{References}

Borjesson, F. and Holtta-Otto, K. (2012). "Improved clustering algorithm for design structure matrix", Proceedings of the ASME 2012 International Design Engineering Technical Conferences and Computers and Information in Engineering Conference, IDETC/CIE 2012; Chicago, IL; United States; August 12-15, 2012, ASME, pp. 921-930. https://doi.org/10.1115/DETC2012-70076

Borjesson, F. and Hölttä-Otto, K. (2014), “A module generation algorithm for product architecture based on component interactions and strategic drivers", Research in Engineering Design, Vol. 25 No. 1, pp. 31-51. https://doi.org/10.1007/s00163-013-0164-2

Booth, J.W. et al. (2017), "The design for additive manufacturing worksheet", Journal of Mechanical Design, Vol. 139 No. 10. https://doi.org/10.1115/1.4037251

Boothroyd, G. (1980), Design for Assembly - A Designer's Handbook, Department of Mechanical Engineering, University of Massachusetts, Amherst.

Boothroyd, G. (1987), "Design for assembly - The key to design for manufacture", The International Journal of Advanced Manufacturing Technology, Vol. 2 No. 3, pp. 3-11. https://doi.org/10.1007/2Fbf02601481

Boothroyd, G. (1996), "Design for Manufacture and Assembly: The Boothroyd-Dewhurst Experience", In: Huang, G.Q. (Ed.), Design for X, Springer, Netherlands, pp. 19-40. https://doi.org/10.1007/2F978-94-0113985-4_2

Cabello, M.J. et al. (2018). "New integrative approach to existing design for assembly (DFA) methodologies: application on elevator components", Proceedings of the DESIGN 2018 15th International Design Conference, Dubrovnik, Croatia, May 21-24, 2018, The Design Society, Glasgow, pp. 215-224. https://doi.org/10.21278/idc.2018.0381

Fournier, C.P. (2018), Claims Management Challenges in the "Modularized" Project Execution Environment, [online] Long International, Inc, Available at: http://www.long-intl.com/articles/Long_Intl_Claims_Mgmt_ Challenges_in_the_Modularized_Proj_Exec_Environment.pdf (accessed 10.10.2019).

Gutierrez, C.I. (1998), Integration analysis of product architecture to support effective team co-location, [Master thesis], Massachusetts Institute of Technology.

Kuo, T.C., Huang, S.H. and Zhang, H.C. (2001), "Design for manufacture and design for "X": Concepts, applications, and perspectives", Computers and Industrial Engineering, Vol. 41 No. 3, pp. 241-260. https://doi.org/10.1016/S0360-8352(01)00045-6 
López, P.H., Avilés, J. and Reboredo, L. (2016), “Aplicación de modularización en proyectos de refinería”, Petrotecnia, pp. 68-73.

Lucas Engineering Systems Ltd. (1993), Design For Manufacture and Assembly Practitioners Manual, University Of Hull.

Masín, I. (2014), “A comparison of DFA Methods for Manual Assembly”, In: Modern Methods of Construction Design Part of the Lecture Notes in Mechanical Engineering book series (LNME), Springer, Switzerland, pp. 265-271. https://doi.org/10.1007/978-3-319-05203-8_38

Mignacca, B. et al. (2018). "We Never Built Small Modular Reactors (SMRs), but What Do We Know About Modularization in Construction?", Proceedings of the 2018 / 26th International Conference on Nuclear Engineering, London, UK, July 22-26, 2018, p. V001T13A012. https://doi.org/10.1115/ICONE26-81604

Nevelius, C. and Deak, L. (2015), Product design changes for automatic assembly: A case study for finding automated assembly solutions, [online] CHALMERS University of Technology, Available at: http://publications.lib.chalmers.se/records/fulltext/218764/218764.pdf (accessed 10.10.2019).

Ou, Z. et al. (2018), "The Practice and Development of Prefabricated Bridges", IOP Conference Series: Materials Science and Engineering, Vol. 392. https://doi.org/10.1088/1757-899X/392/6/062086

Pimmler, T.U. and Eppinger, S.D. (1994). "Integration Analysis of Product Decompositions", Proceedings of the ASME Sixth International Conference on Design Theory and Methodology, Minneapolis, Minnesota, September 11-14.

Poli, C., Dastidar, P. and Graves, R. (1992), "Design knowledge acquisition for DFM methodologies", Research in Engineering Design, Vol. 4 No. 3, pp. 131-145. https://doi.org/10.1007/BF01607942

Price, B., Mahaley, M. and Shimer, W. (2014), "Optimize small-scale LNG production with modular SMR technology", Gas processing, pp. 21-26.

Ramos, A. (2018), Applying design methodologies to the use case of large products, [Master thesis], Universidad del País Vasco UPV-EHU.

Remirez, A. et al. (2019), "New design for assembly methodology adapted to large size products: Application on a solar tracker design", In: Putnik, G.D. (Ed.), Procedia CIRP 84, Elsevier, Portugal, pp. 468-473. https://doi.org/10.1016/j.procir.2019.05.002

Rogers, J.L. (1996), “DeMAID/GA USER'S GUIDE Design Manager's Aid for Intelligent Decomposition with a Genetic Algorithm”, In: Nasa Technical Memorandum, 110241, Hampton, Virginia.

Rossi, F. et al. (2019), "A systematic methodology for the modularization of manufacturing systems during early design”, In: Flexible Services and Manufacturing Journal, Springer, US, pp. 1-44. https://doi.org/10.1007/ s10696-019-09338-7

Sharman, D.M. and Yassine, A.A. (2007), "Architectural valuation using the design structure matrix and real options theory", Concurrent Engineering Research and Applications, Vol. 15 No. 2, pp. 157-173. https://doi.org/10.1177/1063293X07079320

Steyn, J. and Van Heerden, F. (2015), Modularisation in the Process Industry, [online] OTC. https://www. ownerteamconsult.com/wp-content/uploads/2017/02/Insight-Article-019-Modularisation.pdf (accessed 10.10.2019)

Steward, D.V. (1981), "The design structure system: A method for managing the design of complex systems", IEEE Transactions on Engineering Management, Vol. EM-28 No. 3, pp. 71-74. https://doi.org/10.1109/ TEM.1981.6448589

Thebeau, R.E. (2001), Knowledge management of system interfaces and interactions for product development processes, [Master thesis], Massachusetts Institute of Technology.

Wahdan, H.G., Kassem, S.S. and Abdelsalam, H.M. (2016). "A Cuckoo Search clustering algorithm for Design Structure Matrix", Proceedings of the 5th International Conference on Operations Research and Enterprise Systems - ICORES 2016, Rome, Italy, February 23-25, 2016, pp. 36-43. https://doi.org/10.5220/00056 93000360043 